
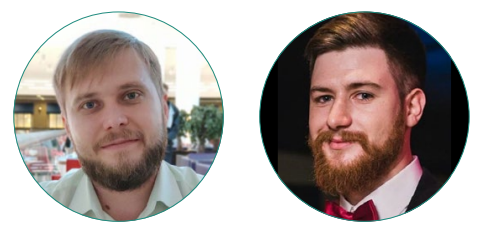

Д. Лемешевский, Л. Р. Грунин

\title{
ОЦЕНКА ОБЪЕМА РЫНКА РЕКЛАМНЫХ INSTAGRAM-РАЗМЕЩЕНИЙ В РФ
}

\section{Правильная ссылка на статью:}

Лемешевский Д., Грунин Л. Р. Оценка объема рынка рекламных Instagram-размещений в РФ // Мониторинг общественного мнения: экономические и социальные перемены. 2021. № 5. C. 185-191. https://doi.org/10.14515/monitoring.2021.5.1987.

\section{For citation:}

Lemeshevskiy D., Grunin L. R. (2021) Assessment of Market Size for the Instagram Ad Market in Russia. Monitoring of Public Opinion: Economic and Social Changes. No. 5. P. 185-191. https://doi.org/10.14515/monitoring.2021.5.1987. (In Russ.) 
ОЦЕНКА ОБЪЕМА РЫНКА РЕКЛАМНЫХ INSTAGRAM-PAЗMЕЩЕНИЙ В РФ

ЛЕМЕШЕВСКИЙ ДМитриЙ - операционный директор, WholsBloger, Москва, Россия

E-MAIL: dlemeshevskiy@whoisblogger.com https://orcid.org/0000-0002-0772-6379

ГРУнИН Лев Романович - генеральный директор, WholsBlogger, Москва, Россия

E-MAIL: Igrunin@whoisblogger.com

https://orcid.org/0000-0002-3229-9890

Аннотация. В статье описан методологический подход к оценке объема рынка рекламных размещений y Instagram-блогеров, а также дан краткий анализ значений, полученных в ходе оценки объема рынка за 2019 и 2020 гг. В рамках методологической части описан поэтапный подход к сбору базы всех публикаций блогеров, выявлению среди них рекламных публикаций, а также оценке стоимости размещения найденных рекламных публикаций. В ходе анализа полученных оценок объема рынка даны комментарии относительно распределению бюджетов по форматам публикаций, масштабу блогеров, а также описание сезонной динамики расходов внутри года.

Ключевые слова: Instagram, маркетинг влияния, инфлюенс-маркетинг, оценка рынка
ASSESSMENT OF MARKET SIZE FOR THE INSTAGRAM AD MARKET IN RUSSIA

Dmitry LEMESHEVSKIY 1 - Chief Operating Officer (COO)

E-MAIL:dlemeshevskiy@whoisblogger.com https://orcid.org/0000-0002-0772-6379

Lev R. GRUNIN ${ }^{1}$ - Chief Executive Officer (CEO)

E-MAIL: Igrunin@whoisblogger.com

https://orcid.org/0000-0002-3229-9890

${ }_{1}$ WholsBloger, Moscow, Russia

Abstract. The article focuses on a methodological approach to assessing the market size of advertising placements for Instagram bloggers. It also analyses the data obtained while evaluating the market size for 2019 and 2020. We describe a step-by-step approach to collecting a database of all bloggers' publications, identifying advertising publications among them, as well as assessing the cost of their placing. We give comments on the distribution of budgets by publication format, the scale of bloggers, and also offer a description of the seasonal dynamics of expenses within a year.

Keywords: Instagram, Influencer marketing, Market assessment

\section{Оценка объема рынка рекламных Instagram-размещений в РФ}

Маркетинг влияния (англ. Influencer marketing) - размещение рекламных интеграций лидерами мнений (блогерами) в социальных сетях - один из наиболее активно растущих сегментов маркетинговых расходов. Глобально данный 
сегмент показывает двузначный рост последние несколько лет, а в абсолютных значениях расходы рекламодателей на интеграции с блогерами достигают оценок 5-10 млрд долл. Российский рынок также находится в стадии интенсивного роста и развития, однако вплоть до 2021 г. не предпринимались попытки системного изучения этого рынка и построения оценки его объема.

Подготовленный в 2021 г. под эгидой IAB Russia (Interactive Advertising Bureau, Ассоциация развития интерактивной рекламы) отчет по российскому рынку Influencer marketing впервые дал комплексную оценку объема рынка, а также темпов его ежегодного роста. Рынок сегментирован по платформам, которые используют блогеры для распространения контента, и включает два крупных сегмента: Instagram и YouTube. Оценка сегмента Instagram была выполнена аналитической компанией WholsBlogger (WIB). В статье детально рассматривается методологический подход к проведению подобных оценок.

\section{Методология исследования}

Укрупненно, задача оценки рынка рекламных размещений у блогеров сводится к трем последовательным шагам.

1. Формирование максимально полной базы всех публикаций, сделанных блогерами на платформе за анализируемый период.

2. Выявление среди всех публикаций рекламных размещений и построение оценки рынка в объемных единицах.

3. Оценка бюджетов найденных публикаций и построение оценки рынка в денежных единицах.

Основной сложностью при реализации первого шага является определение наиболее полного списка всех присутствующих на платформе блогеров, то есть пользователей с высоким охватом аудитории. B WholsBlogger (WIB) мы принимаем, что российским Instagram-блогером считается пользователь, имеющий более 10 тыс. подписчиков, проживающий в России, и при этом аккаунт пользователя не является обезличенным. Таким образом, аккаунты различных компаний, организаций, тематические паблики и проч. не считаются блогерами даже при наличии значительного числа подписчиков.

Для формирования и актуализации списка блогеров в WIB мы используем выборочное исследование подписок выборки реальных россиян - пользователей Instagram. Выборка реальных россиян сформирована за счет уникальной технологии WIB, позволяющей верифицировать реальность пользователя в различных социальных сетях за счет поиска аккаунтов одного и того же человека на различных платформах. Так, если одновременно существуют профили в Instagram и Facebook, совпадающие по публикуемому контенту, либо прямо указывающие друг на друга, либо имеющие в достаточной мере совпадающие социальные графы, то оба профиля признаются принадлежащими одному и тому же реальному человеку. Дополнительно, объединение данных сразу из нескольких профилей позволяет узнать больше информации о человеке и, например, точно определить его принадлежность к российскому сегменту Instagram. Подобный подход дает возможность идентифицировать более 10,5 млн активных пользователей Instagram, имеющих также аккаунты в других социальных сетях. 
Подписки исследуются ежемесячно и на первом этапе фильтруются все аккаунты, обнаруженные в подписках более чем у 0,02\% исследуемой выборки. Затем найденные таким образом аккаунты, которые включают в себя как зарубежных блогеров, так и обезличенные российские аккаунты, подвергаются фильтрации для выявления среди них блогеров. Собранная таким образом выборка блогеров содержит в себе более 50 тыс. активно и регулярно размещающих контент аккаунтов, публикации которых сохраняются для дальнейшего анализа.

На этапе выявления рекламных публикаций из общей массы контента мы используем двухступенчатый подход, подразумевающий на первом этапе определение размещений с признаками рекламы, а на втором этапе - валидацию найденных таким образом размещений. Подобный подход характерен для анализа рынков со слабым регулированием отрасли Influencer marketing, где нет закрепленной регуляторной практики обязательных отметок рекламных публикаций. К таким рынкам относится и российский, где де-факто лишь около $1 \%$ рекламных публикаций помечается блогерами соответствующим образом, например, с помощью хештега \#реклама и т. п. В российской действительности рекламные размещения можно идентифицировать по наличию в них специфических маркеров, связанных напрямую с рекламодателем. Среди таких маркеров - уникальные хештеги вида \#название_бренда, упоминания и отметки в публикациях Instagramаккаунта бренда, а также использование ссылок на сайт бренда.

Сложность, продиктованная реалиями рынка, заключается в том, что для выявления рекламы недостаточно знать набор универсальных маркеров, какими являются, например, упомянутый хештег \#реклама или его английский аналог \#ad. Необходимо знать набор таких маркеров для каждого из брендов, размещающих рекламу у блогеров. Для решения этой задачи в WholsBlogger налажен процесс ежемесячного мониторинга всех публикаций блогеров и анализ всех возникающих в них маркеров - хештегов и упомянутых/отмеченных Instagramпрофилей - на предмет их рекламной функции. Благодаря этому мы ежемесячно узнаем, о чем пишут блогеры и, видя среди упомянутых маркеров те, которые относятся к брендам, фиксируем факт связи бренда с маркером, а также факт размещения брендом рекламы в Instagram. Более чем двухлетний опыт подобной аналитики позволил накопить базу знаний о более чем четырех тысячах брендов и используемых ими маркеров рекламных размещений.

Однако описанный подход сам по себе дает относительно высокую долю ложноположительных срабатываний в тех случаях, когда блогер использует в публикации маркер бренда не в целях его рекламы. Наиболее яркие примеры - отметки аккаунтов брендов одежды на фотографиях, где блогер демонстрирует свой гардероб, которые являются скорее информативными и не преследуют цели продвижения бренда одежды. Поэтому публикации, отфильтрованные по признаку наличия в них маркеров рекламы, необходимо провалидировать на втором этапе для оценки и учета ошибки первого рода. Процесс валидации происходит поэтапно, начиная с применения разработанных эвристических критериев (например, маркеры двух брендов одежды или двух брендов авто в одной публикации с высокой вероятностью говорят о том, что она не является рекламной) и заканчивая выборочной ручной валидацией. Также на этапе валидации публикации группируются 
в зависимости от числа подписчиков их автора. Вследствие этого шага наблюдается очевидная тенденция на снижение ошибки первого рода для более крупных блогеров по сравнению с более мелкими. Крупные авторы значительно реже, чем мелкие, используют маркеры брендов в своих публикациях в нерекламных целях. В анализе размещений за 2020 год на вход валидации было передано более 70 тыс. размещений с наличием маркеров брендов, а по итогам валидации ошибка первого рода была зафиксирована на уровне 25-50\% в зависимости от числа подписчиков блогеров.

Еще один этап валидации полученных результатов - это проверка полноты, или оценка ошибки второго рода. Источником такой ошибки могут быть случаи, когда блогер использовал некорректный (или вовсе не использовал) маркер бренда в рекламной публикации, либо блогер по какой-то причине не попал в список всех блогеров. Для подобной валидации мы используем списки фактических размещений тех рекламных кампаний, аналитику для которых мы проводили. Таким образом, сравнивая достоверный список размещений с размещениями, найденными согласно нашей методологии, можно оценить долю "потерянных" размещений. При анализе размещений за 2020 г. ошибка второго рода была зафиксирована на уровне 5-10\%, также показывая тенденцию на снижение для более крупных блогеров.

Сформировав таким образом выборку рекламных размещений, остается решить последнюю задачу - оценить бюджеты данных размещений. При оценке стоимости рекламного размещения в Instagram учитывается несколько факторов:

- формат размещения (это может быть фото-пост, видео-пост, либо короткоживущая публикация в сториз),

- количество подписчиков у блогера,

- масштаб блогера в целом по числу подписчиков.

Оценка бюджета строится для каждого найденного размещения путем умножения числа подписчиков блогера на среднее значение "стоимости 1 подписчика". Такие значения вычисляются по набору данных о фактической стоимости рекламы у блогеров, которые мы получаем в рамках работы с нашими клиентами. Стоит отметить, что если применять данный подход к отдельно взятым публикациям или выборке публикаций одного автора, то, разумеется, результат может оказаться далеким от реальности. Причина такого расхождения заключается в целом ряде факторов, среди которых можно отметить существенные различия в ценообразовании для разных типов брендов (например, продвижение алкогольного бренда чаще всего обходится дороже, чем продовольственного), хаотичный подход блогеров к ценообразованию, необходимость передачи прав на контент рекламодателю и другие возможные условия размещения. Однако при оценке средней “стоимости 1 подписчика" мы сравнили значения, полученные нами при усреднении значений по собственной базе знаний, со значениями, полученными аналогичным образом другими игроками рынка, и увидели, что они совпадают. Таким образом, можно утверждать, что в среднем по рынку стоимость ${ }^{1}$ размещения, например,

\footnotetext{
1 Здесь и далее под стоимостью понимается стоимость размещения для рекламодателя, которая значительно отличается от гонорара блогера. Поскольку в большинстве случаев блогеры рекрутируются в рекламные кампании через агентства, то стоимость размещения для рекламодателя включает в себя также агентскую комиссию и НДС.
} 
рекламной сториз варьируется в пределах 0,06-0,15 руб. за одного подписчика, а стоимость поста - в пределах 0,16-0,38 руб. за 1 подписчика. Самая низкая стоимость за одного подписчика характерна для самых крупных блогеров, затем она растет по мере снижения охвата блогера, достигая наибольшего значения для блогеров масштаба 50-250 тыс. подписчиков, а затем снова снижается. Такая зависимость объясняется тем, что по мере роста аудитории качество контакта блогера с подписчиками снижается (например, для более крупных блогеров наблюдается в среднем более низкий показатель Engagement Rate, или ER, paвный отношению числа реакций на публикацию к числу подписчиков). Обратное поведение зависимости для самых мелких блогеров обусловлено, скорее всего, недостаточным уровнем профессионализма мелких блогеров (до 50 тыс. подписчиков), что не позволяет им заявлять достаточно высокие стоимости размещений.

В результате, собрав все составляющие воедино, становится возможным построить итоговую оценку рынка Instagram-размещений, которая для 2020 г. составила 12,6 млрд рублей, что на 69\% выше соответствующего значения 2019 г.

\section{Обсуждение результатов}

Полученные результаты позволяют не только оценить рынок в целом, но также проанализировать его в нескольких разрезах. Так, доли бюджетов для размещения долгоживущих (постов) и короткоживущих (сториз) публикаций составляют $56 \%$ и $44 \%$ соответственно. Короткоживущие размещения при этом имеют меньшую стоимость за одного подписчика, что приводит к тому, что совокупный потенциальный охват рекламных сториз превышает охват рекламных постов (см. рис. 1).

Рис. 1 Структура рынка рекламных интеграций Instagram в РФ по форматам, 2020 г.

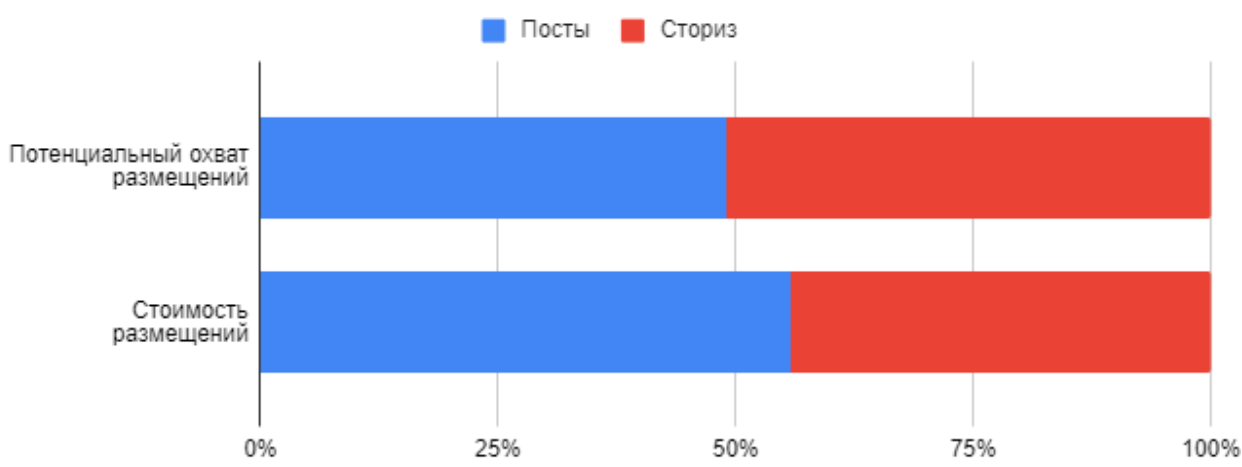

Отдельный интерес представляет распределение бюджетов по масштабу блогеров (см. рис. 2). На наиболее крупных блогеров (более 1 млн подписчиков) приходится $53 \%$ всех бюджетов, в то время как на самых мелких блогеров (до 50 тыс. подписчиков) - лишь 4\%. При этом количество блогеров с более чем 1 млн подписчиков едва приближается к 1 тыс. ( 2\% от общего числа блогеров), а блогеров с 10-50 тыс. подписчиков - около 35 тысяч ( $65 \%$ от общего числа блогеров). 
Рис. 2. Структура рынка рекламных интеграций Instagram в РФ по числу подписчиков блогеров, 2020 г.

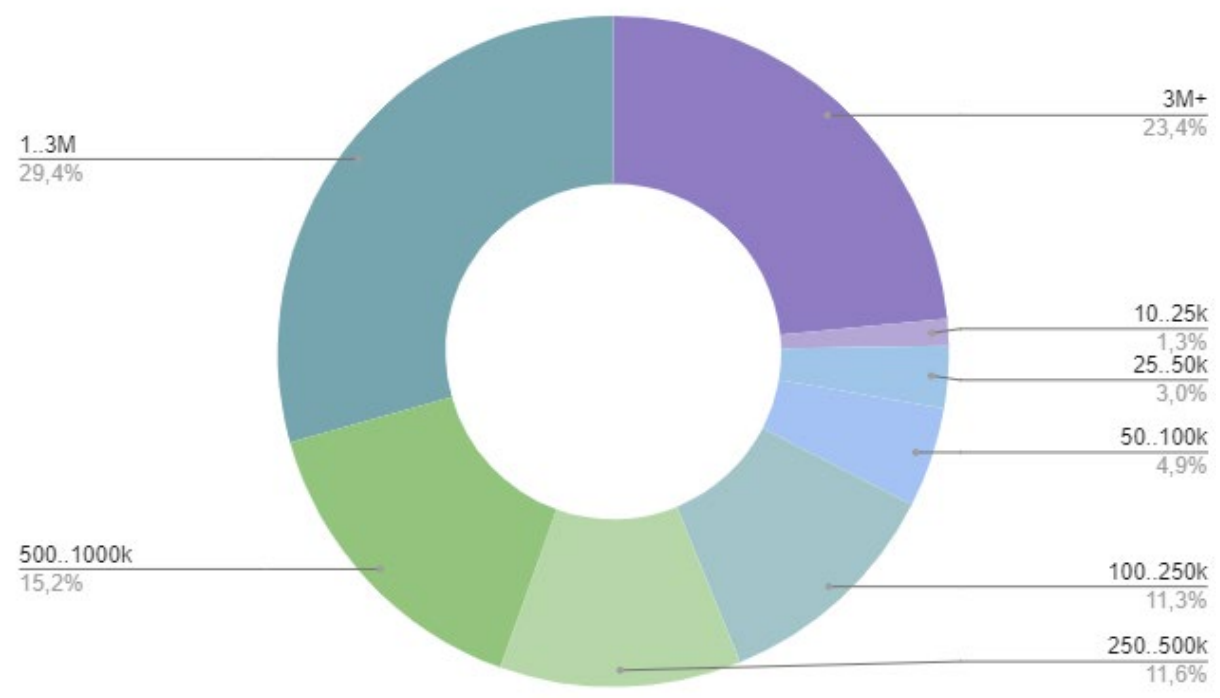

Сравнение поквартальной динамики 2019 и 2020 гг. позволяет оценить эффект пандемии COVID-19 на рынок размещений у блогеров. Если принять, что I квартал 2020 г. не испытал заметного влияния пандемии, то рост год к году составит $+83 \%$. Сравнивая с соответствующими показателями для II-IV кварталов (которые составляют +63-66\%) можно заключить, что пандемия снизила темпы роста рынка на 17-20 процентных пунктов в трех кварталах 2020 г.-это соответствует снижению динамики на 14 процентных пунктов в целом за год, что эквивалентно сокращению рекламных бюджетов на 1,1 млрд рублей относительно “базового" уровня роста год к году I квартала 2020 г. (см. рис. 3).

Рис. З. Анализ бюджетов рекламных интеграций Instagram в РФ

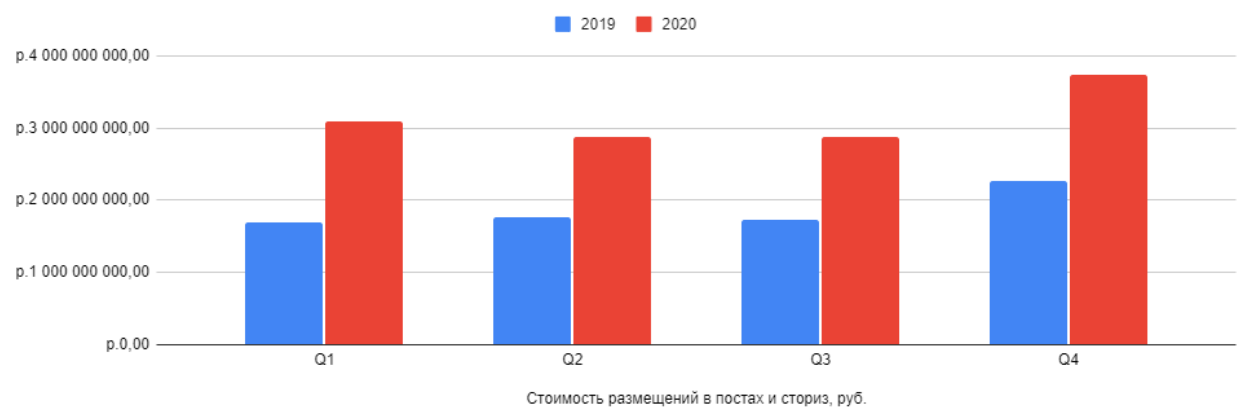

